\title{
PREDICTION OF DYNAMIC CHARACTERISTICS OF VIBRATING STRUCTURE WITH UNCERTAIN PARAMETERS
}

\author{
Imran Ahemad Khan ${ }^{1}$ and G. K. Awari ${ }^{2}$ \\ ${ }^{1}$ Faculty Mechanical Department, \\ Priyadarshini College of Engineering, \\ Nagpur, India. \\ Email: iak20041978@ rediffmail.com \\ ${ }^{2}$ Tulsiramji Gaikwad-Patil College of Engineering and Technology, \\ Nagpur, India. \\ Email: gkawari@ rediffmail.com
}

\begin{abstract}
This paper is based on a study of vibrational responses of different vibrating systems with uncertain parameters. The concept of uncertainty plays an important role in the design of the practical mechanical system. So it becomes important to study its effects on the mechanical system. A large and varied amount of research has been dedicated to developing techniques which predict the dynamic responses of structures in all frequency domains and structures with uncertainty. The structural element selected in this work is the plate. The plate is considered in terms of mass, stiffness and a combination of mass and stiffness uncertainty. The dynamic characteristic of the plate with all uncertain parameters is found by using the Finite Element Method. In this paper modal and harmonic analysis of the plate is done. In modal analysis the natural frequencies and mode shapes of the plate are found. The response of a bare plate is compared with plates for which different uncertain parameters are considered. Similarly, in harmonic analysis the frequency response function of the bare plate and the plates with all uncertain parameters are compared. During comparison, it was found that due to mass uncertainty minor changes in the natural frequency and in mode shape were obtained. However, due to stiffness the natural frequency, mode shape and FRF were drastically changed. Similarly, due to a combination of mass and stiffness uncertainty drastic changes were observed in the plate response. Because of these uncertainties the complete vibrational characteristics were changed. So it becomes important to consider these uncertainties to avoid misinterpretation while designing plates.
\end{abstract}

Keywords: Uncertain Parameters; vibration analysis; modal analysis; harmonic analysis; FEM.

\section{INTRODUCTION}

Predicting the dynamic response of a vibrating system generally involves determining the equations of motion of the structure and solving them in order to find the natural frequencies and mode shapes of the system to give boundary conditions. The natural frequencies and mode shapes can then be used to predict the response due to an applied excitation [1-3]. For more complex systems, the equations of motion can be approximated using various deterministic modelling techniques such as finite element analysis (FEA) and dynamic stiffness techniques [4-10]. These methods are extensively 
used to predict the linear dynamic response of structures in the low frequency region. Energy methods such as Statistical Energy Analysis (SEA) are appropriate dynamic predictive techniques at high frequencies $[11,12]$. In engineering design, it is important to calculate the response quantities such as the displacement, stress, vibration frequencies, and mode shapes of design parameters. The study of mathematical models involves physical and geometric parameters such as mass density $\rho$, elastic modulus $E$, Poisson's ratio $v$, lengths, and cross-section shape characteristics [13]. In many practical engineering applications, these parameters frequently do not have well-defined values due to non-homogeneity of the mass distribution, geometric properties or physical errors, as well as variation arising from assembly and manufacturing processes $[14,15]$. For many dynamic structures it is not possible to know their exact material properties or geometry. This uncertainty can be due to a number of factors such as variation in material properties, structural dimensions or changes in excitation over time. Changes in excitations over time can be caused by wear or fatigue. For example, the wearing of gear teeth or the development of cracks in the teeth can affect the frequency and the amplitude of the harmonic excitations caused by these rotating components. Because of this, it is very important to study and investigate the effect of uncertainty on the overall dynamic characteristics of the structure [16-19].

Vibroacoustic tests were carried out by Kompella and Bernhard [20] on 98 similar vehicles. They measured both airborne and structure-borne transfer paths across the fleet of vehicles and found that both responses varied by up to $20 \mathrm{~dB}$ in the highfrequency range. For many vibratory systems, uncertainty can have a significant effect on the vibrational response even at low frequencies. Wood and Joachim [21], [22] showed that for a four cylinder car the scatter in structure-borne interior noise can vary by as much as $15 \mathrm{~dB}$. Cornish [23] found great variability in the vibroacoustic responses of five identical vehicles across the entire frequency range from $100 \mathrm{~Hz}$ to $400 \mathrm{~Hz}$.In engineering design these uncertainties in material properties, geometric parameters and boundary conditions are often unavoidable and must be considered. This concept of uncertainty plays an important role in the investigation of various engineering problems [13].

The uncertainty concerned in this paper is the variation in material properties such as mass and stiffness. The response of any system with uncertainty will differ from the response of a system model. The level of variation of the response will depend on the degree of uncertainty. At low frequencies, the effect is often negligible, but as the frequency increases, the effect becomes more significant. In this paper modal and harmonic analysis is done to find and compare the response of a bare plate and plates for which all uncertain parameters are considered.

\section{MATHEMATICAL MODELLING}

All the mechanical structures such as machines, vehicles, aircraft, home appliances and civil engineering structures are made up of plate or combination of plates. Plates possess uncertainty during their manufacturing. It becomes necessary to study the vibration of plate with and without uncertainty. Using the Lagrange-Rayleigh-Ritz technique [24], the equations of motion of a dynamic plate in modal space can be derived. Initially a bare plate dynamic is derived and then the addition of different uncertainty in the plate is derived. 


\section{Bare Rectangular Plate}

The simply supported rectangular bare plate (with no structural uncertainty) is considered as shown in Figure 1. The Lagrange-Rayleigh-Ritz technique is applied to a range of dynamic systems in order to examine the natural frequency statistics. Using the Lagrange-Rayleigh-Ritz technique, the equations of motion of a dynamic system in modal space can be derived. This technique is used to obtain the natural frequencies of the bare plate.

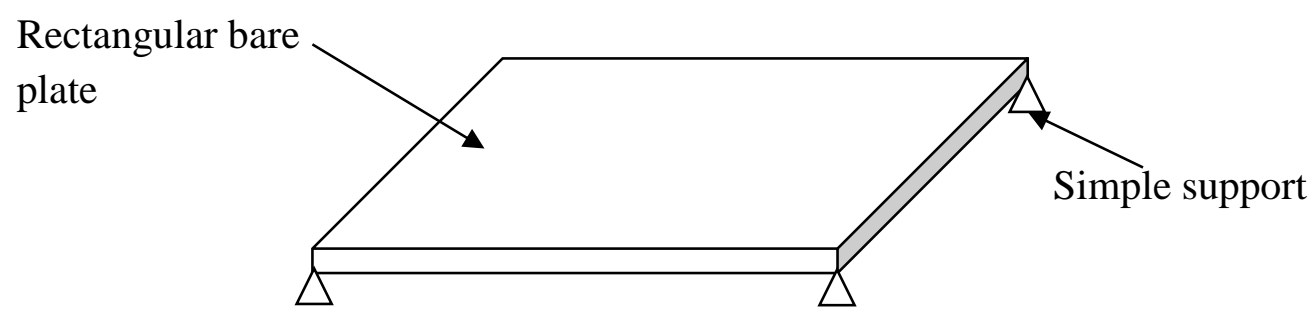

Figure 1. Simply supported rectangular bare plate.

For a simply supported plate the eigen function is:

$$
\phi_{m n}(x)=\phi_{m}(x) \phi_{n}(y)
$$

where $\phi$ is mode shape and $m n$ are mode number.

The sinusoidal mode shapes in the $x$ and $y$ directions, respectively, are described by [24]:

$$
\phi_{m}(x)=\sin \left(m \pi x / L_{x}\right) \text { and } \phi_{n}(y)=\sin \left(n \pi x / L_{y}\right)
$$

where $L_{x}$ and $L_{y}$ are the length of plate in $x$ and $y$ directions, respectively.

The flexural displacement of a bare rectangular plate in modal space is given by [24]

$$
w(x, y, t)=\sum_{m n} q_{m n}(t) \psi_{m n}(x)
$$

where $q$ is the modal coordinate and $m$ and $n$ are the mode numbers of the shape functions in the $x$ and $y$ directions, respectively, and

$$
\psi_{m n}(x)=\psi_{m}(x) \psi_{n}(y)
$$

are the mass-normalised eigen functions which satisfy the following orthogonality condition [25]:

$$
\int_{0}^{L_{x}} \int_{0}^{L_{y}} \rho h \psi_{m n} \psi_{m^{\prime} n^{\prime}} d x d y= \begin{cases}1 & m n=m^{\prime} n^{\prime} \\ 0 & m n \neq m^{\prime} n^{\prime}\end{cases}
$$


$L_{x}$ and $L_{y}$ are respectively the lengths of the plate in the $x$ and $y$ directions, $h$ is the plate thickness and $\rho$ is the density.

For a plate simply supported on all four sides, the mass normalised eigen functions are given by:

$$
\psi_{m n}=\frac{1}{M_{n}} \phi_{m n}(x)=\frac{1}{M_{n}} \sin \left(\frac{m \pi x}{L_{x}}\right) \sin \left(\frac{n \pi y}{L_{y}}\right)
$$

where $M_{n}=\rho h L_{x} L_{y} / 4$ is the modal mass.

Using the orthogonality condition, an expression for the kinetic energy of a bare plate becomes:

$$
\begin{aligned}
& T=\frac{\rho h}{2} \int_{0}^{L_{x}} \int_{0}^{L_{y}} \dot{w}^{2}(x) d x d y \\
& =\frac{\rho h}{2} \sum_{m n} \sum_{j k} \dot{q}_{m n} \dot{q}_{j k} \psi_{m n}(x) \psi_{j k}(x) \\
& =\frac{1}{2} \sum \dot{q}_{m n}{ }^{2}
\end{aligned}
$$

where $\dot{w}$ denotes the derivative of $w$ with respect to time.

Expression for the potential energy of the plate can be obtained as:

$$
V=\frac{1}{2} \sum_{m n} \omega_{m n}^{2} q_{m n}^{2}
$$

where $\omega_{m n}=\sqrt{\frac{D}{\rho h}}\left(\left(\frac{m \pi}{L_{x}}\right)^{2}+\left(\frac{n \pi}{L_{y}}\right)^{2}\right)$

corresponds to the natural frequencies of the bare plate and

$$
D=\frac{E h^{3}}{12\left(1-v^{2}\right)}
$$

is the plate flexural rigidity and $E$ and $v$ are respectively Young's modulus and Poisson's ratio. Lagrange's equation for a particular modal coordinate $j$ is given by [24]:

$$
\frac{d}{d t}\left(\frac{\partial T}{\partial \dot{q}_{j}}\right)-\frac{\partial T}{\partial q_{j}}+\frac{\partial V}{\partial q_{j}}=0, \quad j=1,2 \ldots \ldots N
$$

Differentiating the kinetic and potential energies with respect to the modal coordinate and substituting into Lagrange's equation results in the equation of motion of the bare plate:

$$
\ddot{q}+\omega^{2} q=0
$$


The natural frequencies can then be obtained by eigenvalue analysis [27].

\section{Uncertain Mass Loaded Plate}

Now consider the uncertain mass loaded plate as shown in Figure 2. For this the equation of motion is:

$$
\ddot{q}_{p q}+\sum_{N_{m}} \sum_{m n} m_{a} \ddot{q}_{m n} \psi_{m n}\left(x_{m}\right) \psi_{p q}\left(x_{m}\right)+\omega_{p q}^{2} q_{p q}=0
$$

where $N_{m}$ is the number of point masses, $\psi_{m n}(x)$ are the mass-normalised eigen functions, and $x_{m}$ corresponds to the random locations of the added masses.

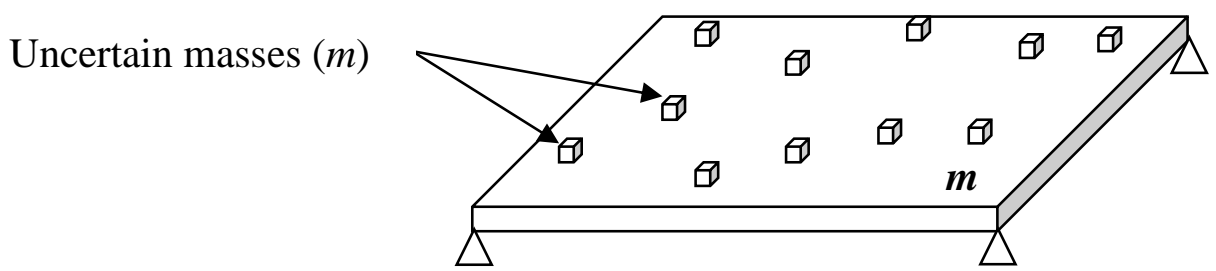

Figure 2. Simply supported rectangular plate with mass uncertainty.

\section{Uncertain Spring (Stiffness) Loaded Plate}

Now consider the uncertain spring loaded plate as shown in Figure 3. For this the equation of motion is:

$$
\ddot{q}_{p q}+\sum_{N_{m}} \sum_{m n} k q_{m n} \psi_{m n}\left(x_{k}\right) \psi_{p q}\left(x_{k}\right)+\omega_{p q}^{2} q_{p q}=0
$$

where $N_{k}$ springs with ground (of stiffness $k$ ) and $\psi_{m n}(x)$ are the mass-normalised eigen functions and $X_{k}$ correspond to the random locations of the added springs

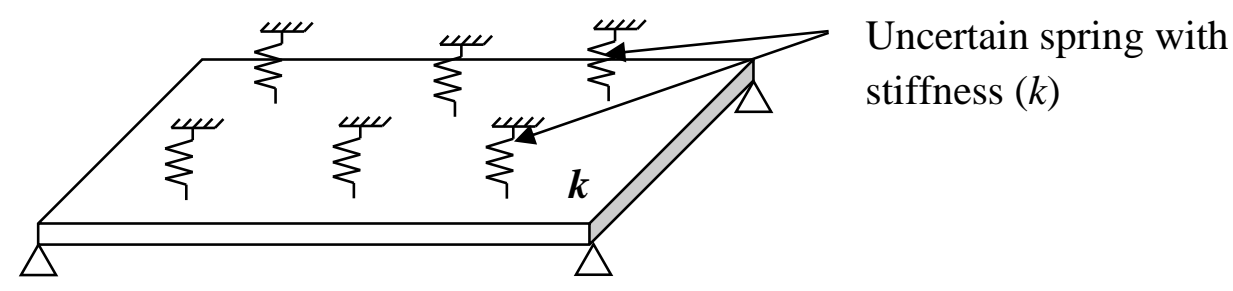

Figure 3. Simply supported rectangular plate with stiffness uncertainty.

\section{Uncertain Mass-and-spring-loaded Plate}

Now consider a mass-and-spring-loaded plate as shown in Figure 4. For this the equation of motion is [16]: 


$$
\ddot{q}_{p q}+\sum_{N_{m}} \sum_{m n} m_{a} \ddot{q}_{m n} \psi_{m n}\left(x_{m}\right) \psi_{p q}\left(x_{m}\right)+\sum_{N_{m}} \sum_{m n} k q_{m n} \psi_{m n}\left(x_{k}\right) \psi_{p q}\left(x_{k}\right)+\omega_{p q}^{2} q_{p q}=0
$$

where $N_{m}$ number of point masses and $N_{k}$ springs to ground (of stiffness $k$ ) and $\psi_{m n}(x)$ are the mass-normalised eigen functions, and $x_{m}$ and $x_{k}$ respectively correspond to the random locations of the added masses and springs.

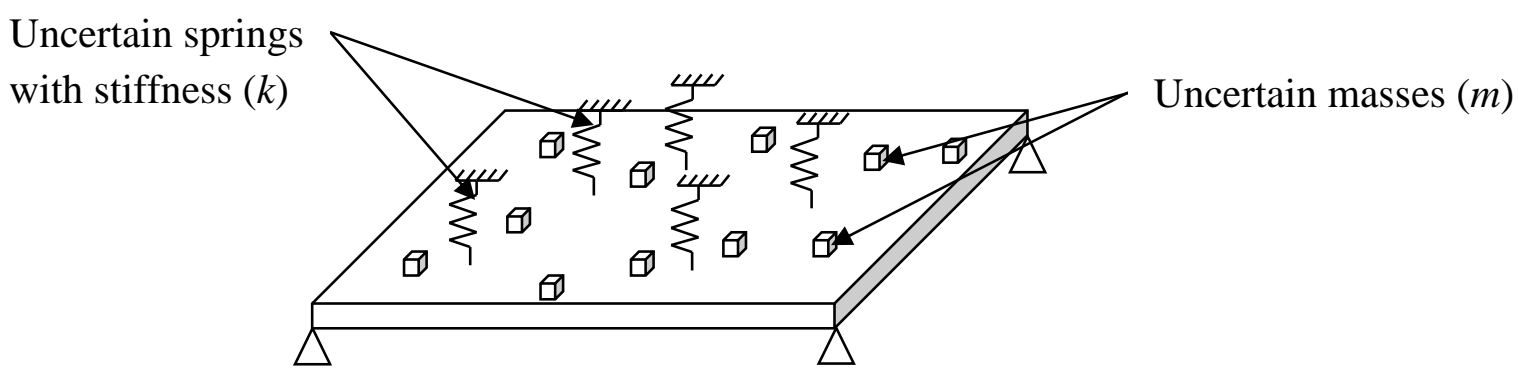

Figure 4. Simply supported rectangular plate with mass and stiffness uncertainty.

\section{MODELLING OF RECTANGULAR PLATE WITH AND WITHOUT UNCERTAITY}

A rectangular plate of dimension $500 \mathrm{~mm} \times 600 \mathrm{~mm} \times 2 \mathrm{~mm}$ of steel material with properties of $\rho=7.86 \times 10^{-9}$ tone $/ \mathrm{mm}^{3}, v=0.3, Y=2 \times 10^{5} \mathrm{MPa}$ was created. All material properties and simply-supported boundary conditions were applied to all edges. The type of element selected is quadratic shell element 181. Shell 181 is suitable for analysing thin to moderately-thick shell structures. It is a four-node element with six degrees of freedom at each node translations in the $x, y$, and $z$ directions, and rotations about the $\mathrm{x}-, \mathrm{y}-$, and $\mathrm{z}$-axis.

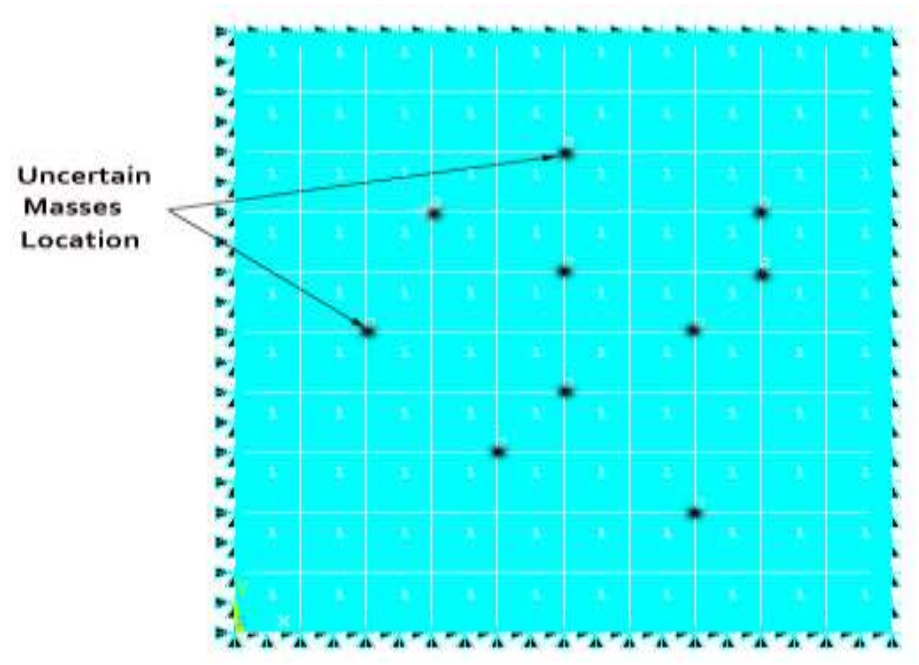

Figure 5. Discrete rectangular plate with 10 uncertain mass elements.

The degenerate triangular option should only be used as filler elements in mesh generation. Shell 181 is well-suited for linear, large rotation, and/or large strain nonlinear applications. The change in shell thickness is accounted for in nonlinear 
analyses [28]. The total mass of the plate is $4.698 \times 10^{-2}$ tons. Mass uncertainty is taken as $2 \%$ of the total mass [1]. The number of masses taken is 10 . All are randomly placed on a plate and located by black spots in Figure 5[4]. The discrete rectangular plate with the location of 10 uncertain mass elements is shown in Figure 5. For uncertain masses 'mass 21 element' is taken which has three degree of freedom at each node [28].

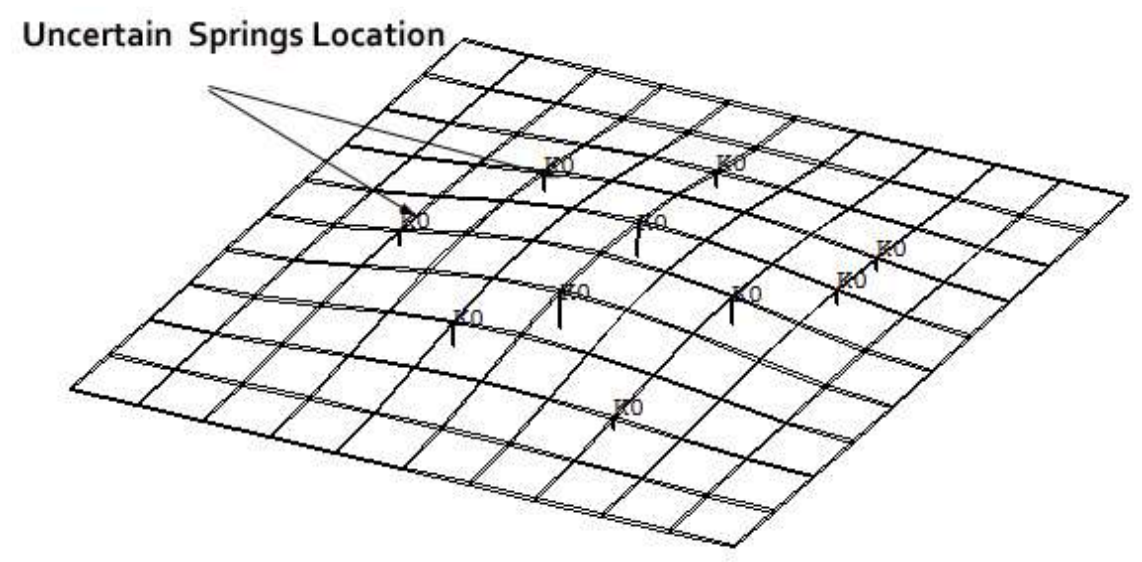

Figure 6. Discrete rectangular plate with 10 uncertain spring elements.

The 10 spring elements are added at the random locations. The locations are the same as the locations of the uncertain masses in Figure 6. The stiffness of each spring is $0.1 \mathrm{~N} / \mathrm{mm}$. The stiffness element used is 'COMBIN14'. It has longitudinal or torsional capability in 1-D, 2-D, or 3-D applications. The longitudinal spring-damper option is a uniaxial tension-compression element with up to three degrees of freedom at each node translation in the nodal $x, y$, and $z$ directions [28].

\section{EFFECT OF UNCERTAIN PARAMETERS ON NATURAL FREQUENCY AND MODE SHAPES}

The frequency range is taken from $0 \mathrm{~Hz}$ to $500 \mathrm{~Hz}$. The plate is excitedin 10 modes. The solver used is Block Lanczos [28]. It gives 10 natural frequency values for the bare plate, mass uncertainty plate, stiffness uncertainty plate and a combination of mass and stiffness uncertainty plates as shown in Table 1 and also in Figure 7 and Figure 8.

Table 1. Natural frequency value for 10 mode shapes.

\begin{tabular}{cccccccc}
\hline $\begin{array}{c}\text { Mode } \\
\text { No. }\end{array}$ & $\begin{array}{c}\text { Bare } \\
\text { plate } \\
(\mathrm{Hz})\end{array}$ & $\begin{array}{c}\text { Mass } \\
\text { uncertain } \\
\text { plate } \\
(\mathrm{Hz})\end{array}$ & $\begin{array}{c}\text { \% decrease in } \\
\text { frequency due } \\
\text { to mass } \\
\text { uncertainty }\end{array}$ & $\begin{array}{c}\text { Stiffness } \\
\text { uncertain } \\
\text { plate } \\
(\mathrm{Hz})\end{array}$ & $\begin{array}{c}\text { \% increase in } \\
\text { frequency } \\
\text { due to } \\
\text { stiffness }\end{array}$ & $\begin{array}{c}\text { Mass and } \\
\text { stiffness } \\
\text { uncertain } \\
\text { plate }(\mathrm{Hz})\end{array}$ & $\begin{array}{c}\text { \% increase in } \\
\text { frequency } \\
\text { due to mass } \\
\text { and stiffness }\end{array}$ \\
\hline $\mathbf{1}$ & 32.481 & 32.413 & 0.21 & 60.39 & 85.92 & 60.29 & 85.62 \\
$\mathbf{2}$ & 72.53 & 72.41 & 0.166 & 110.12 & 51.82 & 109.9 & 51.52 \\
$\mathbf{3}$ & 90.16 & 90 & 0.178 & 136.369 & 51.25 & 136.094 & 50.95 \\
$\mathbf{4}$ & 130.4 & 130.229 & 0.131 & 186.857 & 43.29 & 186.546 & 43.06 \\
$\mathbf{5}$ & 139.479 & 139.272 & 0.149 & 190.913 & 36.87 & 190.575 & 36.63 \\
$\mathbf{6}$ & 186.546 & 186.157 & 0.209 & 253.338 & 35.8 & 252.647 & 35.43 \\
$\mathbf{7}$ & 198.207 & 197.443 & 0.387 & 273.848 & 38.16 & 273.358 & 37.92 \\
$\mathbf{8}$ & 227.385 & 227.076 & 0.136 & 301.768 & 32.71 & 301.227 & 32.47 \\
$\mathbf{9}$ & 234.237 & 233.9 & 0.144 & 309.504 & 32.13 & 308.881 & 31.87 \\
\hline
\end{tabular}




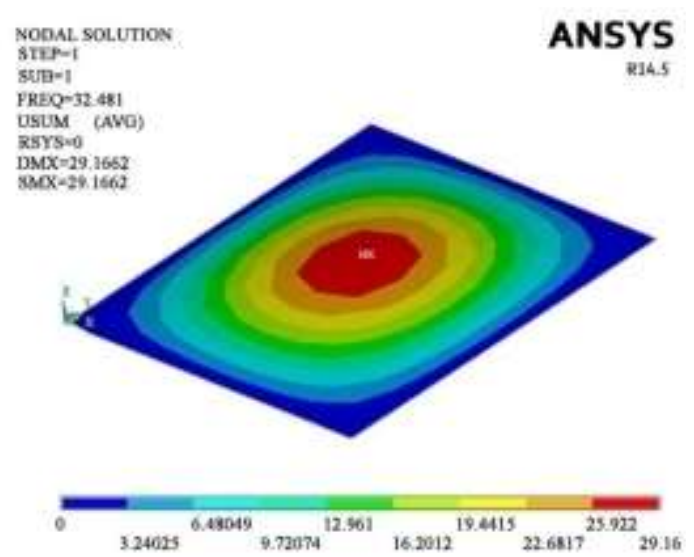

(a)

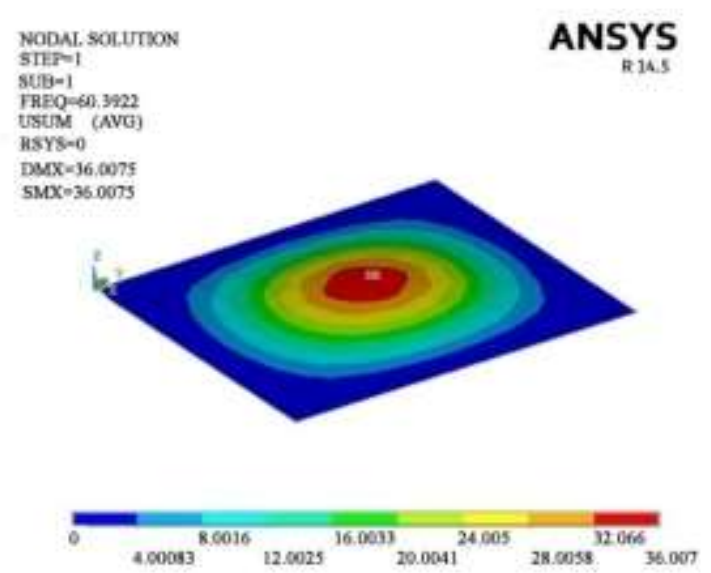

(c)

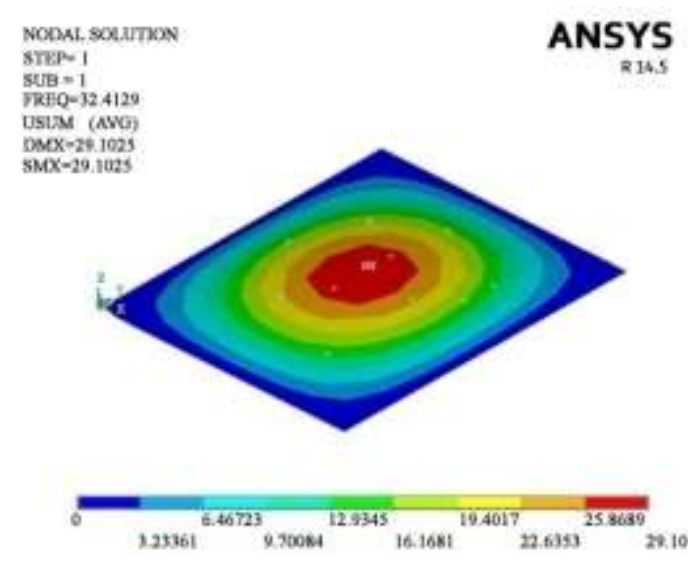

(b)

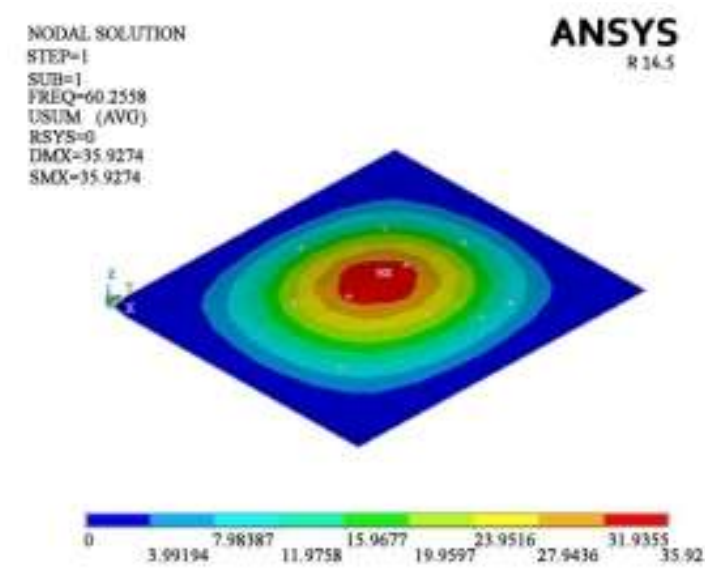

(d)

Figure 7. First mode shape of the plate: (a) bare plate, (b) mass uncertainty, (c) stiffness uncertainty, (d) mass and stiffness uncertainty.

The natural frequency is reduced due to mass uncertainty and its percentage decreased is shown in Table 1. Due to stiffness uncertainty, natural frequency very prominently increases. These are the effects of uncertainty on the natural frequency. In the first mode, there is no change in mode shape obtained, as shown in Figure 7. However, in the second mode, as shown in Figure 8, due to mass uncertainty the mode shape is changed and due to stiffness the phase is changed. These effects of uncertainty on the mode shapes were found.

\section{PREDICTING FREQUENCY RESPONSE FUNCTION}

The frequency response function (FRF) is obtained by harmonic analysis [29]. Harmonic analysis of the plate is done for all edges simply supported. The same plate models are considered with all the uncertain parameters and of the same dimensions, which were considered in modal analysis. In this force vibration study, a force of $1 \mathrm{~N}$ is applied at node location 211, as shown in Figure 9. A frequency range of $0 \mathrm{~Hz}$ to $500 \mathrm{~Hz}$ is given. 


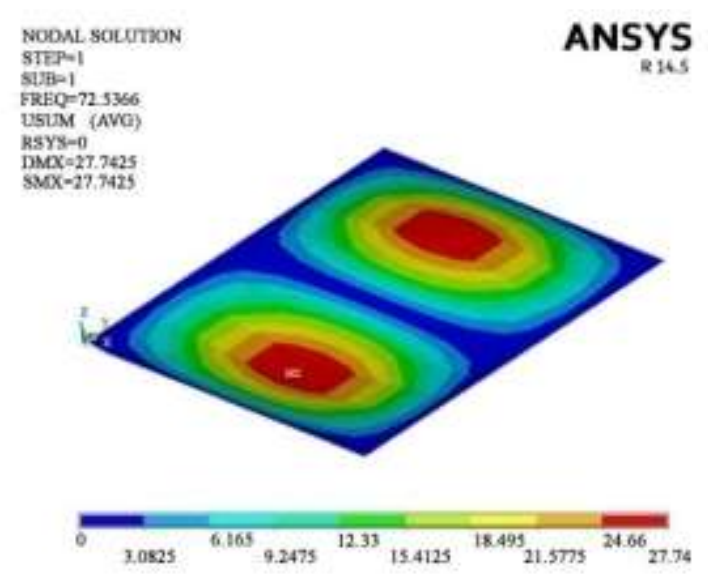

(a)

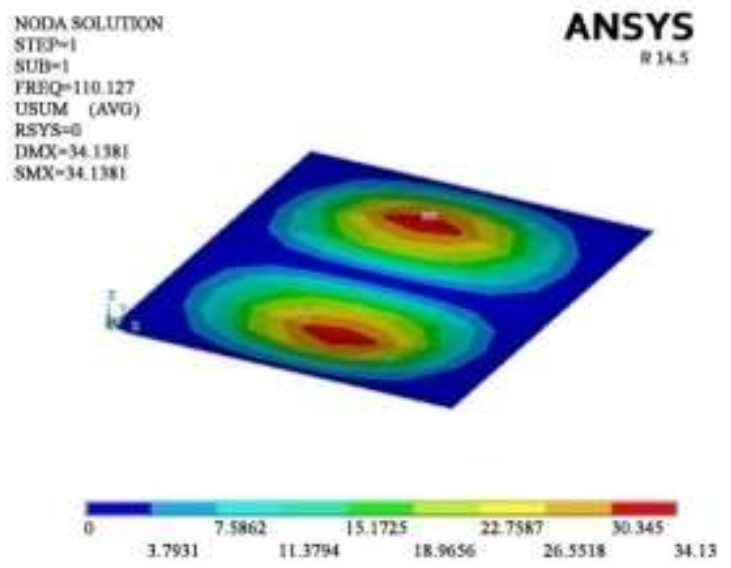

(c)
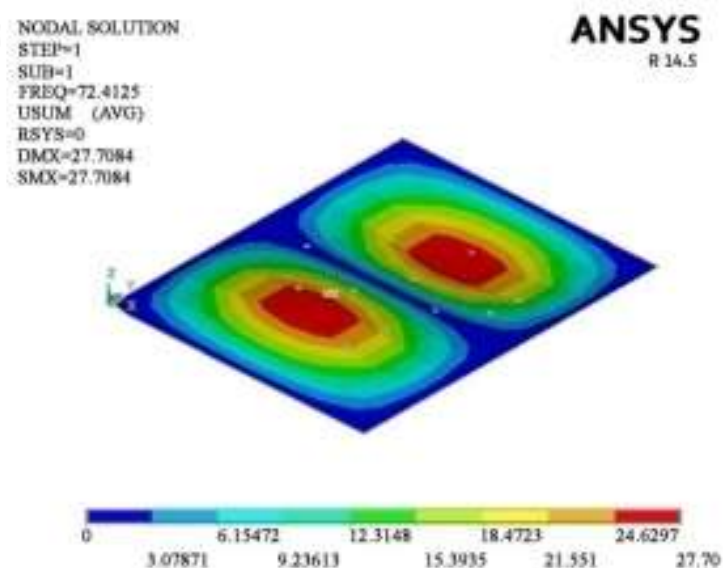

(b)

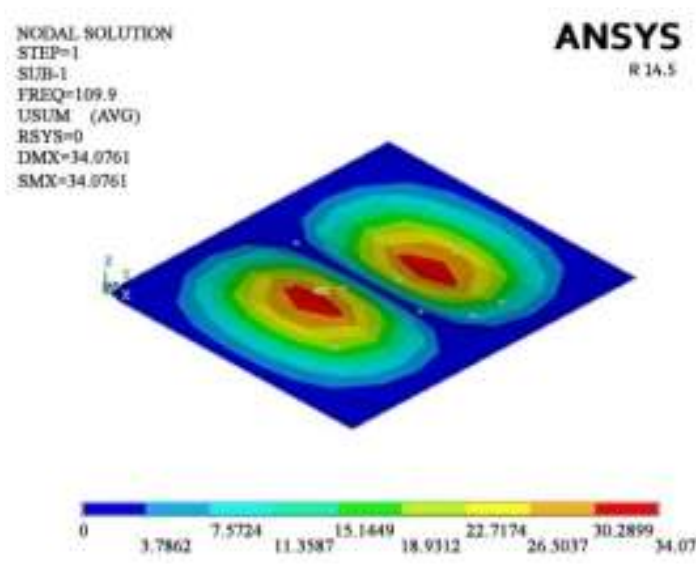

(d)

Figure 8. Second mode shape of the plate: (a) bare plate, (b) mass uncertainty, (c) stiffness uncertainty, (d) mass and stiffness uncertainty.

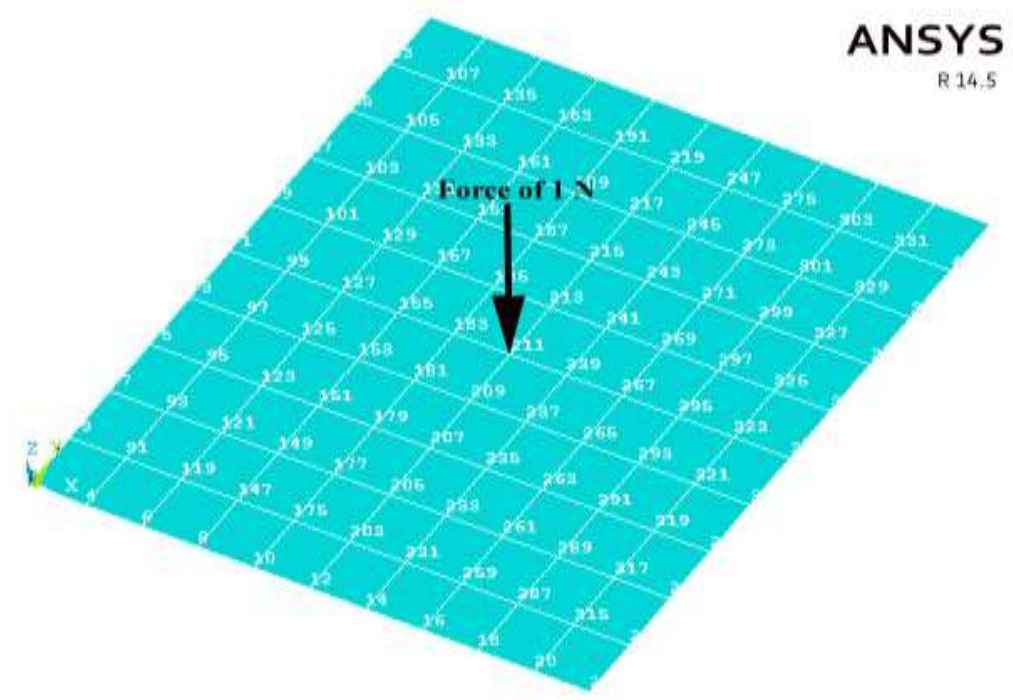

Figure 9. Force loading plate for harmonic analysis. 


\section{Frequency Response Function Analysis}

An FRF was plotted on a linear graph scale. The frequency $(\mathrm{Hz})$ is on the $\mathrm{x}$-axis and amplitude $(\mathrm{mm})$ on the y-axis, as shown in Figure 10. At node number 127 of the plate FRF is taken. The FRF for the bare plate is shown in Figure 10(a) in which first resonance pick occurs at a frequency of $32.481 \mathrm{~Hz}$ and amplitude of $0.41 \mathrm{~mm}$. In Figure 10(b) amplitude is increased by $0.47 \mathrm{~mm}$ because of mass uncertainty at the same frequency. In stiffness uncertainty, Figure 10(c), major changes are observed which indicate that the complete resonance points are shifted to another frequency and their amplitude is also changed. For stiffness and mass uncertainty, Figure 10(d), the frequency of the resonance point is the same, but its amplitude is changed compared with the stiffness uncertainty.

To know the response in more details it is necessary to plot it on a log scale, as shown in Figure 11. This figure is a harmonic response of the plate at node number 127 in the log scale. Here it clearly shows node and antinodes points which are missing in the linear scale (Rao, 2000).Figure 11(a) shows the response of the bare plate which indicates its resonance and anti-resonance point of its different modes. Due to mass uncertainty at frequencies between $400 \mathrm{~Hz}$ and $500 \mathrm{~Hz}$ some different modes of the plate get excited, which was not at the bare plate, as shown in Figure 11(b). Similarly, in stiffness and mass and stiffness uncertainty resonance peaks are shifted to another frequency, the magnitude of the amplitude is also changed, as shown in Figure 11(c, d), compared with the bare plate.

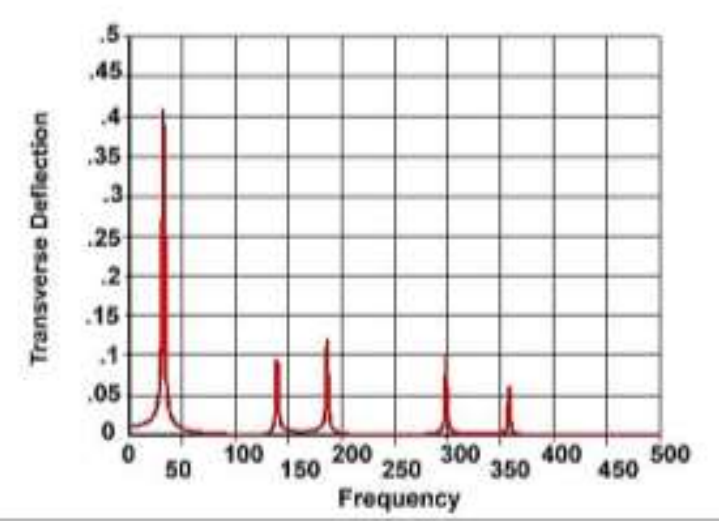

(a)

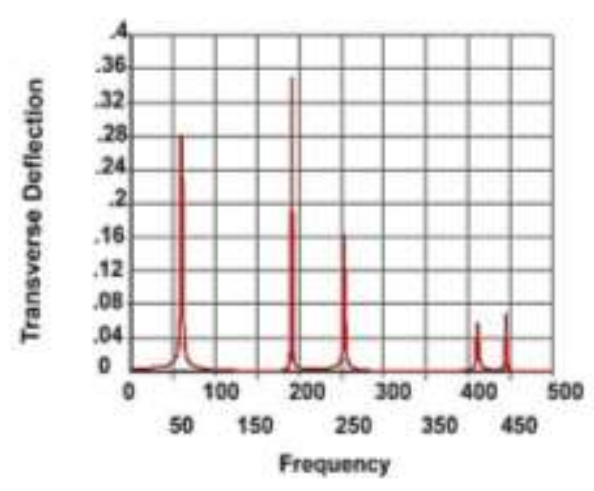

(c)

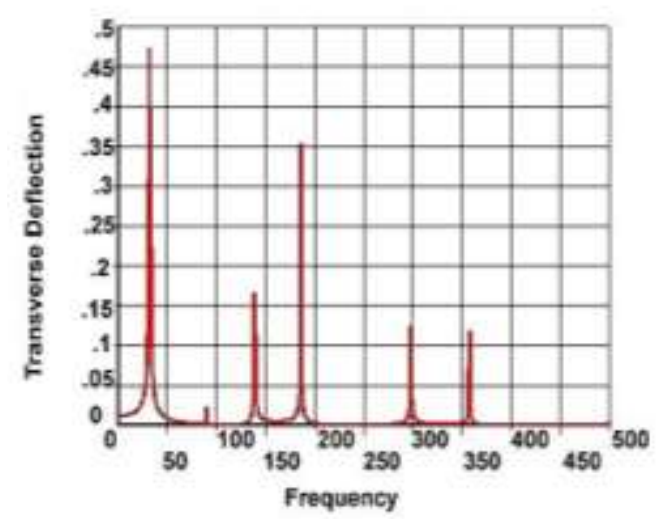

(b)

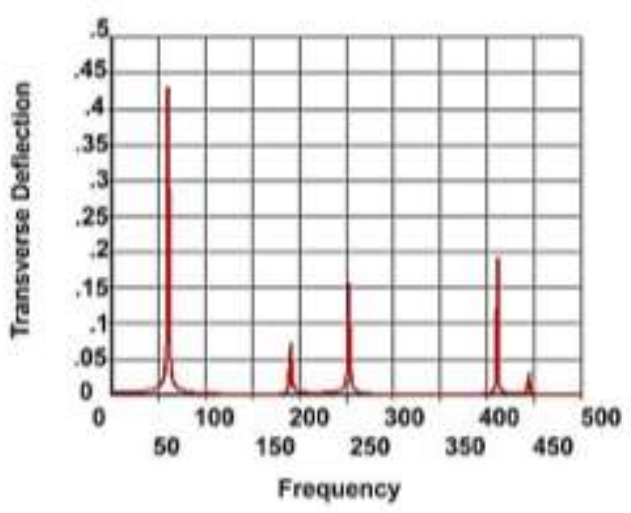

(d)

Figure 10. FRF Plot on Linear Scale: (a) bare plate, (b) mass uncertainty, (c) stiffness uncertainty, (d) mass and stiffness uncertainty. 


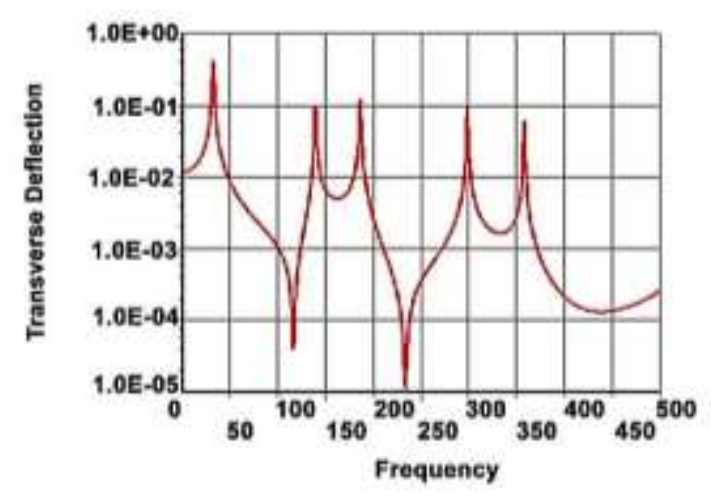

(a)

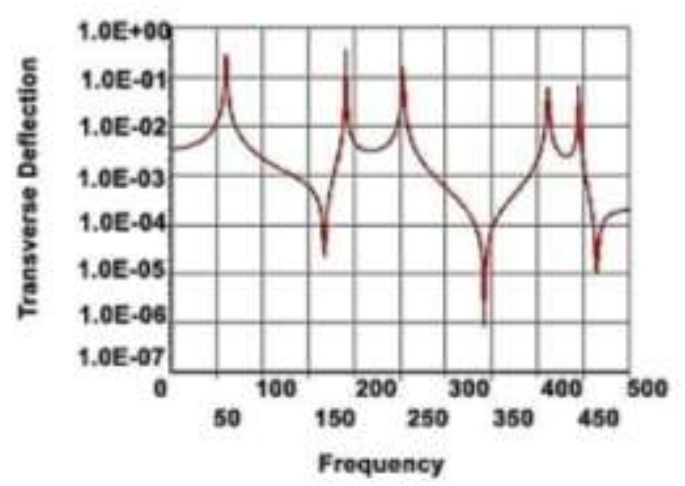

(c)

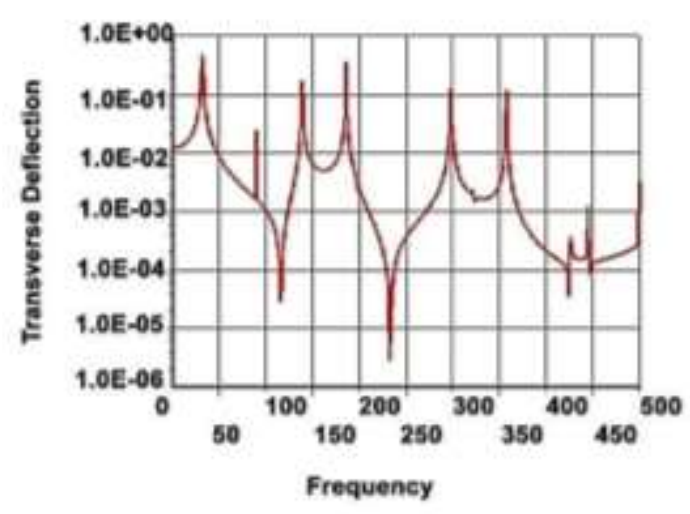

(b)

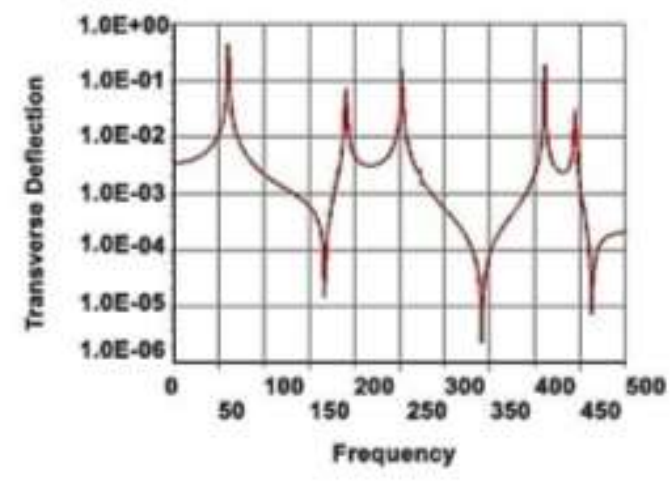

(d)

Figure 11. FRF Plot on Log Scale: (a) bare plate, (b) mass uncertainty, (c) stiffness uncertainty, (d) mass and stiffness uncertainty.

\section{RESULTS AND DISCUSSION}

During the modal analysis of the plate, as shown in Table 1, in the fundamental mode the natural frequency of the plate with mass uncertainty decreased by $0.21 \%$ compared with the bare plate. Due to stiffness uncertainty the natural frequency is drastically increased by $85.92 \%$. Due to mass and stiffness uncertainty the natural frequency is increased by $85.62 \%$. In the harmonic analysis the FRFs are plotted on a linear as well as on a log-log scale. All the FRFs of node 127 for all the parameters of the plate are combined together in Figures 12 and 13 to study the characteristics of the plate effectively. All edges in the simply supported condition plate are excited by an external force and the response of the plate is plotted on the FRF graph, as shown in Figure 12. The frequency of the bare plate in the first fundamental mode (green colour) is $32 \mathrm{~Hz}$ and the amplitude is $0.408448 \mathrm{~mm}$. The mass uncertainty (black colour)is added and the frequency obtained is $32 \mathrm{~Hz}$ and the amplitude is $0.474298 \mathrm{~mm}$. When the stiffness (blue colour) uncertainty is added a very prominent pick with a major shift in frequency of $60 \mathrm{~Hz}$ and large amplitude of $0.281087 \mathrm{~mm}$ is obtained. Due to mass and stiffness uncertainty (red colour)a major frequency shift of $60 \mathrm{~Hz}$ and amplitude of $0.429595 \mathrm{~mm}$ is obtained. The complete resonance point is shifted. 


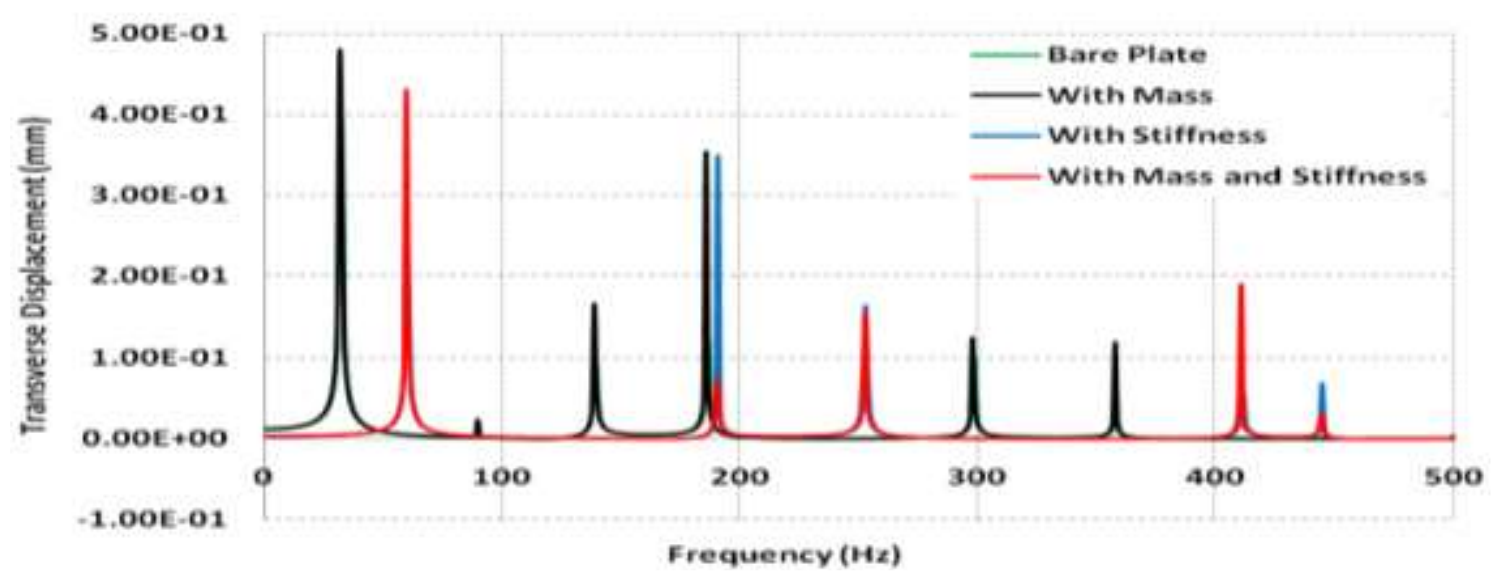

Figure 12. Comparison Linear Plot of Node 127.

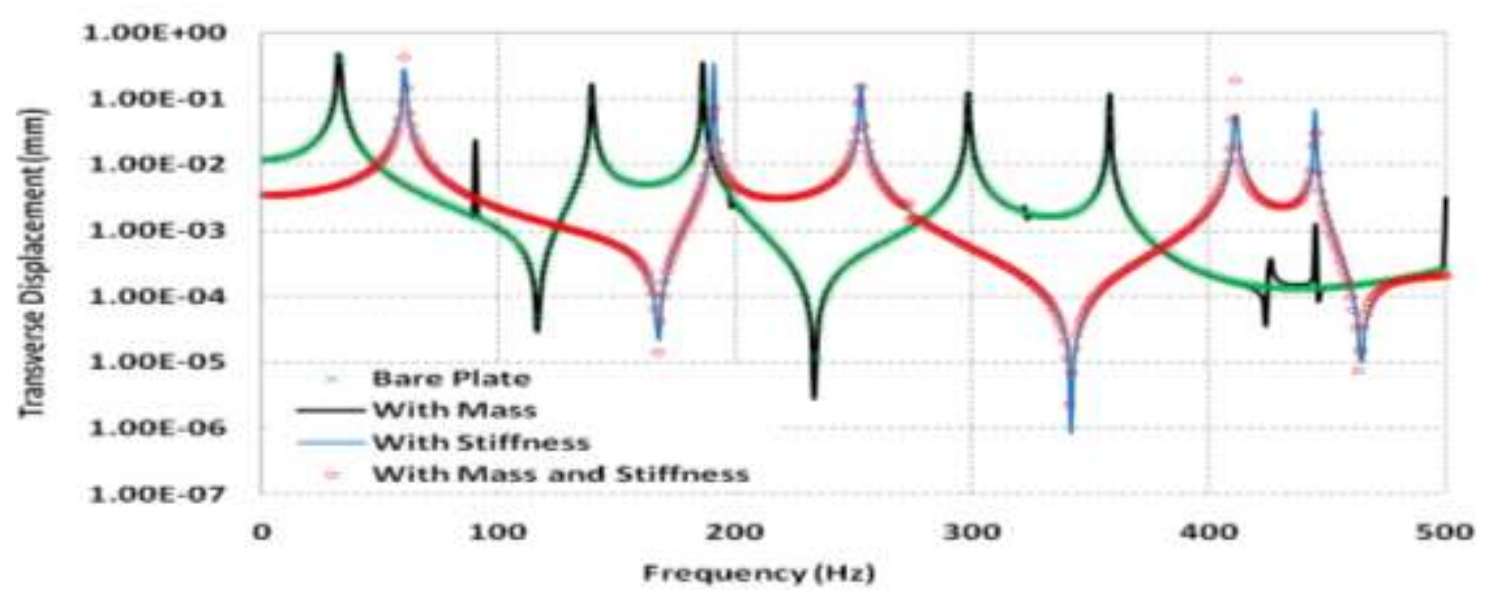

Figure 13. Comparison Log Plot of Node 127.

The FRF of the plate for all edges, simply supported condition is shown in Figure 13. The response of the bare plate is compared with the all other uncertain parameters. In this log-log scale, many details of plate characteristics are studied. In the fundamental mode due to stiffness (blue colour) and a combination of mass and stiffness (red colour) uncertainty, the resonance point is completely shifted compared with the bare plate (green colour). Due to mass uncertainty (black colour) at a high frequency between $50 \mathrm{~Hz}$ and $100 \mathrm{~Hz}$ and $400 \mathrm{~Hz}$ and $450 \mathrm{~Hz}$ different modes of vibration are obtained which were not present in the bare plate characteristic. The completely different characteristics of the plate are obtained due to mass uncertainty.

\section{CONCLUSIONS}

In this analysis four different mass and stiffness uncertain parameters were considered and their effects on vibrational characteristics of the plate were found. During analysis, it was found that due to mass uncertainty very minor changes occurred in the value of natural frequencies. However, due to stiffness uncertainty, it changed dominantly. Due to mass uncertainty mode shape changed and due to stiffness uncertainty its phase was changed. At a high-frequency domain some hidden mode was excited due to a mass uncertainty which was not previously known in the bare plate response. Uncertainty 
affects resonance points due to stiffness uncertainty changes which occur drastically, the complete picks of resonance were shifted and amplitude also changed. Due to mass uncertainty much less change occurred at the resonance points. Because of uncertainty the complete vibrational characteristics were changed. Therefore it becomes important to consider these uncertainties otherwise misinterpretation will occur while designing plates.

\section{ACKNOWLEDGEMENTS}

The authors acknowledge, with tanks to the Department of Mechanical Engineering of Priyadarshini College of Engineering, Nagpur (India) for their support during this research work. Also, thanks to the AICTE, Delhi (India) for funding this research work.

\section{REFERENCES}

[1] Adhikari S, Friswell MI, Lonkar KP. Uncertainty in structural dynamics experimental case studies on beams and plates. ECCOMAS Thematic Conference on Computational Methods in Structural Dynamics and Earthquake Engineering, Rethymno, Crete, Greece2 007. p. 13-6.

[2] Rahman MM, Ariffin AK, Abdullah S. Finite element based vibration fatigue analysis of a new two-stroke linear generator engine component. International Journal of Mechanical and Materials Engineering. 2007;2:63-74.

[3] Rahman MM, Ariffin AK, Jamaludin N, Haron CHC. Vibration fatigue analysis of cylinder head of a new two-stroke free piston engine using finite element approach. SID Structural Integrity and Durability. 2005;1:121-9.

[4] Kessissoglou N, Langley R. Natural frequency statistics of engineering structures. Proceedings of the 11th International Congress on Sound and Vibration, St Petersburg, Russia 2004. p. 5-8.

[5] Rahman MM, Ariffin AK, Abdullah S, Noor MM, Bakar RA, Maleque MA. Assessment of surface treatment on fatigue life of cylinder block for linear engine using frequency response approach. American Journal of Applied Sciences. 2009;6:715-25.

[6] Rahman MM, Ariffin AK, Abdullah S, Rosli AB. Effect of nitriding treatment on fatigue life for free piston linear engine component using frequency response method: A finite element approach. SID Structural Integrity and Durability. 2007;3:197-209.

[7] Rahman MM, Ariffin AK, Jamaludin N, Haron CHC, Bakar RA. Fatigue life prediction of Two-Stroke free piston engine mounting using frequency response approach. European Journal of Scientific Research. 2008;22:480-93.

[8] Rahman MM, Bakar RA, Sani MSM, Noor MM. Investigation into surface treatment on fatigue life for cylinder block of linear engine using frequency response approach. 15th International Congress on Sound and Vibration, 2008. p. 2119-27.

[9] Balcı M, Gündoğdu Ö. Determination of physical properties of laminated composite beam via the inverse vibration problem method. Journal of Mechanical Engineering and Sciences. 2013;5:611-22.

[10] Ab Latif N, M. Rus AZ. Vibration transmissibility study of high density solid waste biopolymer foam. Journal of Mechanical Engineering and Sciences. 2014;6:772-81. 
[11] Cicirello A, Langley R. The analysis of random systems with combined parametric and non-parametric uncertainty models. Proceedings of the International Conference on Noise and Vibration Engineering,(Leuven, 2010)2010.

[12] Patel CB, Gohil PP, Borhade B. Modeling and vibration analysis of road profile measuring system. International Journal of Automotive and Mechanical Engineering. 2010;1:13-28.

[13] Zhang X-M, Ding H. Design optimization for dynamic response of vibration mechanical system with uncertain parameters using convex model. Journal of Sound and Vibration. 2008;318:406-15.

[14] Manson G. Calculating frequency response functions for uncertain systems using complex affine analysis. Journal of Sound and Vibration. 2005;288:487521.

[15] Jamil N, Yusoff AR, Mansor MH. Literature review of electromagnetic actuator force generation for dynamic modal testing applications. Journal of Mechanical Engineering and Sciences. 2012;3:311-9.

[16] Kessissoglou NJ, Lucas GI. Gaussian orthogonal ensemble spacing statistics and the statistical overlap factor applied to dynamic systems. Journal of Sound and Vibration. 2009;324:1039-66.

[17] Hemin MM, Rahman MM, Omar RM. Dynamic analysis of vehicle arm based on finite element approach. Journal of Advanced Science and Engineering Research. 2011;1:124-36.

[18] Sani MSM, Rahman MM, Noor MM, Kadirgama K, Izham MHN. Identification of dynamics modal parameter for car chassis. IOP Conference Series: Materials Science and Engineering. 2011;17:1-8.

[19] Kamal M, Rahman MM. Study on the dynamic behavior of wishbone suspension system. IOP Conference Series: Materials Science and Engineering. 2012;36:1-8.

[20] Kompella MS, Bernhard RJ. Variation of structural-acoustic characteristics of automotive vehicles. Noise Control Engineering Journal. 1996;44(2):93-99.

[21] Wood L, Joachim C. Scatter of structureborne noise in four cylinder motor vehicles. SAE Technical Paper; 1986.

[22] Wood L, Joachim C. Interior noise scatter in four-cylinder sedans and wagons. International Journal of Vehicle Design. 1987;8:428-438.

[23] Cornish R. A novel approach to optimizing and stabilizing interior noise quality in vehicles. Proceedings of the Institution of Mechanical Engineers, Part D: Journal of Automobile Engineering. 2000;214:685-92.

[24] Meirovitch L. Elements of vibration analysis. McGraw-Hill: New York; 1975.

[25] Timoshenko S, Woinowsky-Krieger S, Woinowsky-Krieger S. Theory of plates and shells: McGraw-Hill: New York; 1959.

[26] Manning R, Ewing, J. (2009). RACQ vehicles technologies. temperatures in cars survey. RACQ vehicles technologies. 2009:1-21.

[27] Soedel W. Vibrations of shells and plates: CRC Press; 2004.

[28] ANSYS®. Structural analysis guide. In: Mechanical A, editor. Help Ansys: ANSYS, Inc; 2013.

[29] Fu ZF, He J. Modal analysis: Butterworth-Heinemann: New York; 2001. 